\title{
LYAPUNOV FUNCTIONALS CONSTRUCTION \\ FOR STOCHASTIC DIFFERENCE SECOND-KIND \\ VOLTERRA EQUATIONS WITH CONTINUOUS TIME
}

\author{
LEONID SHAIKHET
}

Received 4 August 2003

The general method of Lyapunov functionals construction which was developed during the last decade for stability investigation of stochastic differential equations with aftereffect and stochastic difference equations is considered. It is shown that after some modification of the basic Lyapunov-type theorem, this method can be successfully used also for stochastic difference Volterra equations with continuous time usable in mathematical models. The theoretical results are illustrated by numerical calculations.

\section{Stability theorem}

Construction of Lyapunov functionals is usually used for investigation of stability of hereditary systems which are described by functional differential equations or Volterra equations and have numerous applications $[3,4,8,21]$. The general method of Lyapunov functionals construction for stability investigation of hereditary systems was proposed and developed (see $[2,5,6,7,9,10,11,12,13,17,18,19]$ ) for both stochastic differential equations with aftereffect and stochastic difference equations. Here it is shown that after some modification of the basic Lyapunov-type stability theorem, this method can also be used for stochastic difference Volterra equations with continuous time, which are popular enough in researches $[1,14,15,16,20]$.

Let $\{\Omega, \mathfrak{F}, \mathbf{P}\}$ be a probability space, $\left\{\mathfrak{F}_{t}, t \geq t_{0}\right\}$ a nondecreasing family of sub- $\sigma$ algebras of $\mathfrak{F}$, that is, $\mathfrak{F}_{t_{1}} \subset \mathfrak{F}_{t_{2}}$ for $t_{1}<t_{2}$, and $H$ a space of $\mathfrak{F}_{t}$-measurable functions $x(t) \in \mathbb{R}^{n}, t \geq t_{0}$, with norms

$$
\|x\|^{2}=\sup _{t \geq t_{0}} \mathbf{E}|x(t)|^{2}, \quad\|x\|_{1}^{2}=\sup _{t \in\left[t_{0}, t_{0}+h_{0}\right]} \mathbf{E}|x(t)|^{2} .
$$

Consider the stochastic difference equation

$$
x\left(t+h_{0}\right)=\eta\left(t+h_{0}\right)+F\left(t, x(t), x\left(t-h_{1}\right), x\left(t-h_{2}\right), \ldots\right), \quad t>t_{0}-h_{0},
$$


with the initial condition

$$
x(\theta)=\phi(\theta), \quad \theta \in \Theta=\left[t_{0}-h_{0}-\max _{j \geq 1} h_{j}, t_{0}\right] .
$$

Here, $\eta \in H, h_{0}, h_{1}, \ldots$ are positive constants, and $\phi(\theta), \theta \in \Theta$, is an $\mathfrak{F}_{t_{0}}$-measurable function such that

$$
\|\phi\|_{0}^{2}=\sup _{\theta \in \Theta} \mathbf{E}|\phi(\theta)|^{2}<\infty,
$$

the functional $F \in \mathbb{R}^{n}$ satisfies the condition

$$
\left|F\left(t, x_{0}, x_{1}, x_{2}, \ldots\right)\right|^{2} \leq \sum_{j=0}^{\infty} a_{j}\left|x_{j}\right|^{2}, \quad A=\sum_{j=0}^{\infty} a_{j}<\infty .
$$

A solution of problem (1.2), (1.3) is an $\mathfrak{F}_{t}$-measurable process $x(t)=x\left(t ; t_{0}, \phi\right)$, which is equal to the initial function $\phi(t)$ from (1.3) for $t \leq t_{0}$ and with probability 1 defined by (1.2) for $t>t_{0}$.

Definition 1.1. A function $x(t)$ from $H$ is called

(i) uniformly mean square bounded if $\|x\|^{2}<\infty$;

(ii) asymptotically mean square trivial if

$$
\lim _{t \rightarrow \infty} \mathbf{E}|x(t)|^{2}=0
$$

(iii) asymptotically mean square quasitrivial if, for each $t \geq t_{0}$,

$$
\lim _{j \rightarrow \infty} \mathbf{E}\left|x\left(t+j h_{0}\right)\right|^{2}=0 ;
$$

(iv) uniformly mean square summable if

$$
\sup _{t \geq t_{0}} \sum_{j=0}^{\infty} \mathbf{E}\left|x\left(t+j h_{0}\right)\right|^{2}<\infty
$$

(v) mean square integrable if

$$
\int_{t_{0}}^{\infty} \mathbf{E}|x(t)|^{2} d t<\infty
$$

Remark 1.2. It is easy to see that if the function $x(t)$ is uniformly mean square summable, then it is uniformly mean square bounded and asymptotically mean square quasitrivial.

Remark 1.3. It is evident that condition (1.7) follows from (1.6), but the inverse statement is not true. The corresponding function is considered in Example 5.1.

Together with (1.2) we will consider the auxiliary difference equation

$$
x\left(t+h_{0}\right)=F\left(t, x(t), x\left(t-h_{1}\right), x\left(t-h_{2}\right), \ldots\right), \quad t>t_{0}-h_{0},
$$

with initial condition (1.3) and the functional $F$, satisfying condition (1.5). 
Definition 1.4. The trivial solution of (1.10) is called

(i) mean square stable if, for any $\epsilon>0$ and $t_{0} \geq 0$, there exists a $\delta=\delta\left(\epsilon, t_{0}\right)>0$ such that $\|x(t)\|^{2}<\epsilon$ if $\|\phi\|_{0}^{2}<\delta$;

(ii) asymptotically mean square stable if it is mean square stable and for each initial function $\phi$, condition (1.6) holds;

(iii) asymptotically mean square quasistable if it is mean square stable and for each initial function $\phi$ and each $t \in\left[t_{0}, t_{0}+h_{0}\right)$, condition (1.7) holds.

THeOREM 1.5. Let the process $\eta(t)$ in (1.2) satisfy the condition $\|\eta\|_{1}^{2}<\infty$, and there exist a nonnegative functional

$$
V(t)=V\left(t, x(t), x\left(t-h_{1}\right), x\left(t-h_{2}\right), \ldots\right),
$$

positive numbers $c_{1}, c_{2}$, and nonnegative function $\gamma(t)$, such that

$$
\begin{gathered}
\hat{\gamma}=\sup _{s \in\left[t_{0}, t_{0}+h_{0}\right)} \sum_{j=0}^{\infty} \gamma\left(s+j h_{0}\right)<\infty, \\
\mathbf{E} V(t) \leq c_{1} \sup _{s \leq t} \mathbf{E}|x(s)|^{2}, \quad t \in\left[t_{0}, t_{0}+h_{0}\right), \\
\mathbf{E} \Delta V(t) \leq-c_{2} \mathbf{E}|x(t)|^{2}+\gamma(t), \quad t \geq t_{0},
\end{gathered}
$$

where

$$
\Delta V(t)=V\left(t+h_{0}\right)-V(t)
$$

Then the solution of (1.2), (1.3) is uniformly mean square summable.

Proof. Rewrite condition (1.14) in the form

$$
\mathrm{E} \Delta V\left(t+j h_{0}\right) \leq-c_{2} \mathbf{E}\left|x\left(t+j h_{0}\right)\right|^{2}+\gamma\left(t+j h_{0}\right), \quad t \geq t_{0}, j=0,1, \ldots
$$

Summing this inequality from $j=0$ to $j=i$, by virtue of (1.15), we obtain

$$
\mathbf{E} V\left(t+(i+1) h_{0}\right)-\mathbf{E} V(t) \leq-c_{2} \sum_{j=0}^{i} \mathbf{E}\left|x\left(t+j h_{0}\right)\right|^{2}+\sum_{j=0}^{i} \gamma\left(t+j h_{0}\right)
$$

Therefore,

$$
c_{2} \sum_{j=0}^{\infty} \mathbf{E}\left|x\left(t+j h_{0}\right)\right|^{2} \leq \mathbf{E} V(t)+\sum_{j=0}^{\infty} \gamma\left(t+j h_{0}\right), \quad t \geq t_{0} .
$$


We show that the right-hand side of inequality (1.18) is bounded. Really, using (1.14), (1.15), for $t \geq t_{0}$, we have

$$
\begin{aligned}
\mathbf{E} V(t) & \leq \mathbf{E} V\left(t-h_{0}\right)+\gamma\left(t-h_{0}\right) \\
& \leq \mathbf{E} V\left(t-2 h_{0}\right)+\gamma\left(t-2 h_{0}\right)+\gamma\left(t-h_{0}\right) \\
& \leq \cdots \leq \mathbf{E} V\left(t-i h_{0}\right)+\sum_{j=1}^{i} \gamma\left(t-j h_{0}\right) \\
& \leq \cdots \leq \mathbf{E} V(s)+\sum_{j=1}^{\tau} \gamma\left(t-j h_{0}\right),
\end{aligned}
$$

where

$$
s=t-\tau h_{0} \in\left[t_{0}, t_{0}+h_{0}\right), \quad \tau=\left[\frac{t-t_{0}}{h_{0}}\right]
$$

$[t]$ is the integer part of a number $t$.

Since $t=s+\tau h_{0}$, then

$$
\begin{aligned}
& \sum_{j=0}^{\infty} \gamma\left(t+j h_{0}\right)=\sum_{j=0}^{\infty} \gamma\left(s+(\tau+j) h_{0}\right)=\sum_{j=\tau}^{\infty} \gamma\left(s+j h_{0}\right), \\
& \sum_{j=1}^{\tau} \gamma\left(t-j h_{0}\right)=\sum_{j=1}^{\tau} \gamma\left(s+(\tau-j) h_{0}\right)=\sum_{j=0}^{\tau-1} \gamma\left(s+j h_{0}\right) .
\end{aligned}
$$

Therefore, using (1.12), we obtain

$$
\sum_{j=0}^{\infty} \gamma\left(t+j h_{0}\right)+\sum_{j=1}^{\tau} \gamma\left(t-j h_{0}\right)=\sum_{j=0}^{\infty} \gamma\left(s+j h_{0}\right) \leq \hat{\gamma}
$$

So, from (1.18), (1.19), and (1.22), it follows that

$$
c_{2} \sum_{j=0}^{\infty} \mathbf{E}\left|x\left(t+j h_{0}\right)\right|^{2} \leq \hat{\gamma}+\mathbf{E} V(s), \quad t \geq t_{0}, s=t-\left[\frac{t-t_{0}}{h_{0}}\right], h_{0} \in\left[t_{0}, t_{0}+h_{0}\right) .
$$

Using (1.13), we get

$$
\sup _{s \in\left[t_{0}, t_{0}+h_{0}\right)} \mathbf{E} V(s) \leq c_{1} \sup _{t \leq t_{0}+h_{0}} \mathbf{E}|x(t)|^{2} \leq c_{1}\left[\|\phi\|_{0}^{2}+\|x\|_{1}^{2}\right] .
$$


From (1.2), (1.3), and (1.5), for $t \in\left[t_{0}, t_{0}+h_{0}\right]$, we obtain

$$
\begin{aligned}
\mathbf{E}|x(t)|^{2} & =\mathbf{E}\left|\eta(t)+F\left(t-h_{0}, x\left(t-h_{0}\right), x\left(t-h_{0}-h_{1}\right), x\left(t-h_{0}-h_{2}\right), \ldots\right)\right|^{2} \\
& \leq 2\left[\mathbf{E}|\eta(t)|^{2}+\mathbf{E}\left|F\left(t-h_{0}, x\left(t-h_{0}\right), x\left(t-h_{0}-h_{1}\right), x\left(t-h_{0}-h_{2}\right), \ldots\right)\right|^{2}\right] \\
& \leq 2\left[\mathbf{E}|\eta(t)|^{2}+a_{0} \mathbf{E}\left|\phi\left(t-h_{0}\right)\right|^{2}+\sum_{j=1}^{\infty} a_{j} \mathbf{E}\left|\phi\left(t-h_{0}-h_{j}\right)\right|^{2}\right] \\
& \leq 2\left[\|\eta\|_{1}^{2}+A\|\phi\|_{0}^{2}\right] .
\end{aligned}
$$

Using (1.23), (1.24), and (1.25), we have

$$
c_{2} \sum_{j=0}^{\infty} \mathbf{E}\left|x\left(t+j h_{0}\right)\right|^{2} \leq \hat{\gamma}+c_{1}\left[(1+2 A)\|\phi\|_{0}^{2}+2\|\eta\|_{1}^{2}\right] .
$$

From here and (1.8), it follows that the solution of (1.2), (1.3) is uniformly mean square summable. The theorem is proven.

Remark 1.6. Replace condition (1.12) in Theorem 1.5 by the condition

$$
\int_{t_{0}}^{\infty} \gamma(t) d t<\infty
$$

Then the solution of (1.2) for each initial function (1.3) is mean square integrable. Really, integrating (1.14) from $t=t_{0}$ to $t=T$, by virtue of (1.15), we have

$$
\int_{t_{0}}^{T} \mathbf{E}\left(V\left(t+h_{0}\right)-V(t)\right) d t \leq-c_{2} \int_{t_{0}}^{T} \mathbf{E}|x(t)|^{2} d t+\int_{t_{0}}^{T} \gamma(t) d t
$$

or

$$
\int_{T}^{T+h_{0}} \mathbf{E} V(t) d t-\int_{t_{0}}^{t_{0}+h_{0}} \mathbf{E} V(t) d t \leq-c_{2} \int_{t_{0}}^{T} \mathbf{E}|x(t)|^{2} d t+\int_{t_{0}}^{T} \gamma(t) d t
$$

From here and (1.24), (1.25), it follows that

$$
\begin{aligned}
c_{2} \int_{t_{0}}^{T} \mathbf{E}|x(t)|^{2} d t & \leq \int_{t_{0}}^{t_{0}+h_{0}} \mathbf{E} V(t) d t+\int_{t_{0}}^{T} \gamma(t) d t \\
& \leq c_{1} h_{0}\left[(1+2 A)\|\phi\|_{0}^{2}+2\|\eta\|_{1}^{2}\right]+\int_{t_{0}}^{\infty} \gamma(t) d t<\infty,
\end{aligned}
$$

and by $T \rightarrow \infty$, we obtain (1.9).

Remark 1.7. Suppose that for (1.10) the conditions of Theorem 1.5 hold with $\gamma(t) \equiv 0$. Then the trivial solution of (1.10) is asymptotically mean square quasistable. Really, in the case $\gamma(t) \equiv 0$ from inequality (1.26) for (1.10) $(\eta(t) \equiv 0)$, it follows that $c_{2} \mathbf{E}|x(t)|^{2} \leq$ $c_{1}(1+2 A)\|\phi\|_{0}^{2}$ and condition (1.7) follows. It means that the trivial solution of (1.10) is asymptotically mean square quasistable. 
From Theorem 1.5 and Remark 1.6, it follows that an investigation of the solution of (1.2) can be reduced to the construction of appropriate Lyapunov functionals. Below, some formal procedure of Lyapunov functionals construction for (1.2) is described.

Remark 1.8. Suppose that in (1.2) $h_{0}=h>0, h_{j}=j h, j=1,2, \ldots$. Putting $t=t_{0}+s h$, $y(s)=x\left(t_{0}+s h\right)$, and $\xi(s)=\eta\left(t_{0}+s h\right)$, one can reduce $(1.2)$ to the form

$$
\begin{gathered}
y(s+1)=\xi(s+1)+G(s, y(s), y(s-1), y(s-2), \ldots), \quad s>-1, \\
y(\theta)=\phi(\theta), \quad \theta \leq 0 .
\end{gathered}
$$

Below, the equation of type (1.31) is considered.

\section{Formal procedure of Lyapunov functionals construction}

The proposed procedure of Lyapunov functionals construction consists of the following four steps.

Step 1. Represent the functional $F$ at the right-hand side of (1.2) in the form

$$
F\left(t, x(t), x\left(t-h_{1}\right), x\left(t-h_{2}\right), \ldots\right)=F_{1}(t)+F_{2}(t)+\Delta F_{3}(t)
$$

where

$$
\begin{gathered}
F_{1}(t)=F_{1}\left(t, x(t), x\left(t-h_{1}\right), \ldots, x\left(t-h_{k}\right)\right), \\
F_{j}(t)=F_{j}\left(t, x(t), x\left(t-h_{1}\right), x\left(t-h_{2}\right), \ldots\right), \quad j=2,3, \\
F_{1}(t, 0, \ldots, 0) \equiv F_{2}(t, 0,0, \ldots) \equiv F_{3}(t, 0,0, \ldots) \equiv 0,
\end{gathered}
$$

$k \geq 0$ is a given integer, $\Delta F_{3}(t)=F_{3}\left(t+h_{0}\right)-F_{3}(t)$.

Step 2. Suppose that for the auxiliary equation

$$
y\left(t+h_{0}\right)=F_{1}\left(t, y(t), y\left(t-h_{1}\right), \ldots, y\left(t-h_{k}\right)\right), \quad t>t_{0}-h_{0},
$$

there exists a Lyapunov functional

$$
v(t)=v\left(t, y(t), y\left(t-h_{1}\right), \ldots, y\left(t-h_{k}\right)\right),
$$

which satisfies the conditions of Theorem 1.5.

Step 3. Consider Lyapunov functional $V(t)$ for (1.2) in the form $V(t)=V_{1}(t)+V_{2}(t)$, where the main component is

$$
V_{1}(t)=v\left(t, x(t)-F_{3}(t), x\left(t-h_{1}\right), \ldots, x\left(t-h_{k}\right)\right) .
$$

Calculate $\mathrm{E} \Delta V_{1}(t)$ and, in a reasonable way, estimate it.

Step 4. In order to satisfy the conditions of Theorem 1.5 , the additional component $V_{2}(t)$ is chosen by some standard way. 


\section{Linear Volterra equations with constant coefficients}

We demonstrate the formal procedure of Lyapunov functionals construction described above for stability investigation of the scalar equation

$$
\begin{gathered}
x(t+1)=\eta(t+1)+\sum_{j=0}^{[t]+r} a_{j} x(t-j), \quad t>-1, \\
x(s)=\phi(s), \quad s \in[-(r+1), 0],
\end{gathered}
$$

where $r \geq 0$ is a given integer, $a_{j}$ are known constants, and the process $\eta(t)$ is uniformly mean square summable.

3.1. The first way of Lyapunov functionals construction. Following the procedure, represent (Step 1) equation (3.1) in the form (2.1) with $F_{3}(t)=0$,

$$
F_{1}(t)=\sum_{j=0}^{k} a_{j} x(t-j), \quad F_{2}(t)=\sum_{j=k+1}^{[t]+r} a_{j} x(t-j), \quad k \geq 0,
$$

and consider (Step 2) the auxiliary equation

$$
\begin{aligned}
y(t+1) & =\sum_{j=0}^{k} a_{j} y(t-j), \quad t>-1, k \geq 0, \\
y(s) & = \begin{cases}\phi(s), & s \in[-(r+1), 0], \\
0, & s<-(r+1) .\end{cases}
\end{aligned}
$$

Take into consideration the vector $Y(t)=(y(t-k), \ldots, y(t-1), y(t))^{\prime}$ and represent the auxiliary equation (3.3) in the form

$$
Y(t+1)=A Y(t), \quad A=\left(\begin{array}{cccccc}
0 & 1 & 0 & \cdots & 0 & 0 \\
0 & 0 & 1 & \cdots & 0 & 0 \\
\vdots & \vdots & \vdots & \vdots & \vdots & \vdots \\
0 & 0 & 0 & \cdots & 0 & 1 \\
a_{k} & a_{k-1} & a_{k-2} & \cdots & a_{1} & a_{0}
\end{array}\right)
$$

Consider the matrix equation

$$
A^{\prime} D A-D=-U, \quad U=\left(\begin{array}{cccc}
0 & \cdots & 0 & 0 \\
\vdots & \vdots & \vdots & \vdots \\
0 & \cdots & 0 & 0 \\
0 & \cdots & 0 & 1
\end{array}\right) \text {, }
$$

and suppose that the solution $D$ of this equation is a positive semidefinite symmetric matrix of dimension $k+1$ with the elements $d_{i j}$ such that the condition $d_{k+1, k+1}>0$ holds. In 
74 Difference Volterra equations with continuous time

this case the function $v(t)=Y^{\prime}(t) D Y(t)$ is a Lyapunov function for (3.4), that is, it satisfies the conditions of Theorem 1.5, in particular, condition (1.14) with $\gamma(t)=0$. Really, using (3.4), we have

$$
\begin{aligned}
\Delta v(t) & =Y^{\prime}(t+1) D Y(t+1)-Y^{\prime}(t) D y(t) \\
& =Y^{\prime}(t)\left[A^{\prime} D A-D\right] Y(t)=-Y^{\prime}(t) U Y(t)=-y^{2}(t) .
\end{aligned}
$$

Following Step 3 of the procedure, we will construct a Lyapunov functional $V(t)$ for (3.1) in the form $V(t)=V_{1}(t)+V_{2}(t)$, where

$$
V_{1}(t)=X^{\prime}(t) D X(t), \quad X(t)=(x(t-k), \ldots, x(t-1), x(t))^{\prime} .
$$

Rewrite now (3.1) using representation (3.2) as

$$
\begin{gathered}
X(t+1)=A X(t)+B(t), \\
B(t)=(0, \ldots, 0, b(t))^{\prime}, \quad b(t)=\eta(t+1)+F_{2}(t),
\end{gathered}
$$

where the matrix $A$ is defined by (3.4). Calculating $\Delta V_{1}(t)$, by virtue of (3.8), we have

$$
\begin{aligned}
\Delta V_{1}(t) & =X^{\prime}(t+1) D X(t+1)-X^{\prime}(t) D X(t) \\
& =(A X(t)+B(t))^{\prime} D(A X(t)+B(t))-X^{\prime}(t) D X(t) \\
& =-x^{2}(t)+B^{\prime}(t) D B(t)+2 B^{\prime}(t) D A X(t) .
\end{aligned}
$$

Put

$$
\alpha_{l}=\sum_{j=l}^{\infty}\left|a_{j}\right|, \quad l=0,1, \ldots
$$

Using (3.8), (3.2), (3.10), and $\mu>0$, we obtain

$$
\begin{aligned}
\mathbf{E} B^{\prime}(t) & D B(t) \\
= & d_{k+1, k+1} \mathbf{E} b^{2}(t)=d_{k+1, k+1} \mathbf{E}\left[\eta(t+1)+F_{2}(t)\right]^{2} \\
\leq & d_{k+1, k+1}\left[(1+\mu) \mathbf{E}|\eta(t+1)|^{2}+\left(1+\mu^{-1}\right) \mathbf{E} F_{2}^{2}(t)\right] \\
= & d_{k+1, k+1}\left[(1+\mu) \mathbf{E}|\eta(t+1)|^{2}+\left(1+\mu^{-1}\right) \mathbf{E}\left(\sum_{j=k+1}^{[t]+r} a_{j} x(t-j)\right)^{2}\right] \\
\leq & d_{k+1, k+1}\left[(1+\mu) \mathbf{E}|\eta(t+1)|^{2}+\left(1+\mu^{-1}\right) \alpha_{k+1} \sum_{j=k+1}^{[t]+r}\left|a_{j}\right| \mathbf{E} x^{2}(t-j)\right] .
\end{aligned}
$$


Since

$$
D B(t)=b(t)\left(\begin{array}{c}
d_{1, k+1} \\
d_{2, k+1} \\
\vdots \\
d_{k, k+1} \\
d_{k+1, k+1}
\end{array}\right), \quad A X(t)=\left(\begin{array}{c}
x(t-k+1) \\
x(t-k+2) \\
\vdots \\
x(t) \\
\sum_{m=0}^{k} a_{m} x(t-m)
\end{array}\right)
$$

then

$$
\begin{aligned}
\mathbf{E} B^{\prime}(t) & D A X(t) \\
= & \mathbf{E} b(t)\left[\sum_{l=1}^{k} d_{l, k+1} x(t-k+l)+d_{k+1, k+1} \sum_{m=0}^{k} a_{m} x(t-m)\right] \\
= & \mathbf{E} b(t)\left[\sum_{m=0}^{k-1}\left(a_{m} d_{k+1, k+1}+d_{k-m, k+1}\right) x(t-m)+a_{k} d_{k+1, k+1} x(t-k)\right] \\
= & d_{k+1, k+1} \sum_{m=0}^{k} Q_{k m} \mathbf{E} b(t) x(t-m),
\end{aligned}
$$

where

$$
Q_{k m}=a_{m}+\frac{d_{k-m, k+1}}{d_{k+1, k+1}}, \quad m=0, \ldots, k-1, \quad Q_{k k}=a_{k} .
$$

Note that

$$
\sum_{m=0}^{k} Q_{k m} \mathbf{E} b(t) x(t-m)=\sum_{m=0}^{k} Q_{k m} \mathbf{E} \eta(t+1) x(t-m)+\mathbf{E} F_{2}(t) \sum_{m=0}^{k} Q_{k m} x(t-m),
$$

and for $\mu>0$,

$$
2|\mathbf{E} \eta(t+1) x(t-m)| \leq \mu \mathbf{E} \eta^{2}(t+1)+\mu^{-1} \mathbf{E} x^{2}(t-m) .
$$

\section{Putting}

$$
\beta_{k}=\sum_{m=0}^{k}\left|Q_{k m}\right|=\left|a_{k}\right|+\sum_{m=0}^{k-1}\left|a_{m}+\frac{d_{k-m, k+1}}{d_{k+1, k+1}}\right|
$$


76 Difference Volterra equations with continuous time

and using (3.2), (3.10), and (3.17), we obtain

$$
\begin{aligned}
2 \mathbf{E} F_{2}( & t) \sum_{m=0}^{k} Q_{k m} x(t-m) \\
& =2 \sum_{m=0}^{k} \sum_{j=k+1}^{[t]+r} Q_{k m} a_{j} \mathbf{E} x(t-m) x(t-j) \\
& \leq \sum_{m=0}^{k} \sum_{j=k+1}^{[t]+r}\left|Q_{k m}\right|\left|a_{j}\right|\left(\mathbf{E} x^{2}(t-m)+\mathbf{E} x^{2}(t-j)\right) \\
& \leq \sum_{m=0}^{k}\left|Q_{k m}\right|\left(\alpha_{k+1} \mathbf{E} x^{2}(t-m)+\sum_{j=k+1}^{[t]+r}\left|a_{j}\right| \mathbf{E} x^{2}(t-j)\right) \\
& =\alpha_{k+1} \sum_{m=0}^{k}\left|Q_{k m}\right| \mathbf{E} x^{2}(t-m)+\beta_{k} \sum_{j=k+1}^{[t]+r}\left|a_{j}\right| \mathbf{E} x^{2}(t-j) .
\end{aligned}
$$

So,

$$
\begin{aligned}
2 \mathbf{E} B^{\prime}(t) D A X(t) \leq d_{k+1, k+1}[ & \beta_{k} \sum_{j=k+1}^{[t]+r}\left|a_{j}\right| \mathbf{E} x^{2}(t-j)+\beta_{k} \mu \mathbf{E} \eta^{2}(t+1) \\
& \left.+\left(\alpha_{k+1}+\mu^{-1}\right) \sum_{m=0}^{k}\left|Q_{k m}\right| \mathbf{E} x^{2}(t-m)\right] .
\end{aligned}
$$

From (3.9), (3.11), and (3.19), we have

$$
\begin{aligned}
\mathbf{E} \Delta V_{1}(t) \leq- & \mathbf{E} x^{2}(t)+d_{k+1, k+1} \\
\times & {\left[\left(\left(1+\mu^{-1}\right) \alpha_{k+1}+\beta_{k}\right) \sum_{j=k+1}^{[t]+r}\left|a_{j}\right| \mathbf{E} x^{2}(t-j)\right.} \\
& \left.+\left(1+\mu\left(1+\beta_{k}\right)\right) \mathbf{E} \eta^{2}(t+1)+\left(\alpha_{k+1}+\mu^{-1}\right) \sum_{m=0}^{k}\left|Q_{k m}\right| \mathbf{E} x^{2}(t-m)\right] .
\end{aligned}
$$

Put now

$$
R_{k m}= \begin{cases}\left(\alpha_{k+1}+\mu^{-1}\right)\left|Q_{k m}\right|, & 0 \leq m \leq k \\ \left(\left(1+\mu^{-1}\right) \alpha_{k+1}+\beta_{k}\right)\left|a_{m}\right|, & m>k\end{cases}
$$

Then (3.20) takes the form

$$
\mathbf{E} \Delta V_{1}(t) \leq-\mathbf{E} x^{2}(t)+d_{k+1, k+1}\left[\left(1+\mu\left(1+\beta_{k}\right)\right) \mathbf{E} \eta^{2}(t+1)+\sum_{m=0}^{[t]+r} R_{k m} \mathbf{E} x^{2}(t-m)\right]
$$


Choose now (Step 4) the functional $V_{2}(t)$ in the form

$$
V_{2}(t)=d_{k+1, k+1} \sum_{m=1}^{[t]+r} q_{m} x^{2}(t-m), \quad q_{m}=\sum_{j=m}^{\infty} R_{k j} .
$$

Then

$$
\begin{aligned}
\Delta V_{2}(t) & =d_{k+1, k+1}\left(\sum_{m=1}^{[t]+1+r} q_{m} x^{2}(t+1-m)-\sum_{m=1}^{[t]+r} q_{m} x^{2}(t-m)\right) \\
& =d_{k+1, k+1}\left(\sum_{m=0}^{[t]+r} q_{m+1} x^{2}(t-m)-\sum_{m=1}^{[t]+r} q_{m} x^{2}(t-m)\right) \\
& =d_{k+1, k+1}\left(q_{1} x^{2}(t)-\sum_{m=1}^{[t]+r} R_{k m} x^{2}(t-m)\right) .
\end{aligned}
$$

From (3.22), (3.24), for the functional $V(t)=V_{1}(t)+V_{2}(t)$, we have

$$
\mathbf{E} \Delta V(t) \leq-\left(1-q_{0} d_{k+1, k+1}\right) \mathbf{E} x^{2}(t)+\gamma(t)
$$

where

$$
\gamma(t)=d_{k+1, k+1}\left(1+\mu\left(1+\beta_{k}\right)\right) \mathbf{E} \eta^{2}(t+1)
$$

Since the process $\eta(t)$ is uniformly mean square summable, then the function $\gamma(t)$ satisfies condition (1.12). So, if

$$
q_{0} d_{k+1, k+1}<1
$$

then the functional $V(t)$ satisfies condition (1.14). It is easy to check that condition (1.13) holds too. Really, using (3.7), (3.23) for the functional $V(t)=V_{1}(t)+V_{2}(t)$ and $t \in[0,1)$, we have

$$
\begin{aligned}
\mathbf{E} V(t) & \leq\|D\| \sum_{j=0}^{k} \mathbf{E} x^{2}(t-j)+d_{k+1, k+1} \sum_{m=1}^{r} q_{m} \mathbf{E} x^{2}(t-m) \\
& \leq\left((k+1)\|D\|+d_{k+1, k+1} \sum_{m=1}^{r} q_{m}\right) \sup _{s \leq t} \mathbf{E} x^{2}(s) .
\end{aligned}
$$

So, if condition (3.27) holds, then the solution of (3.1) is uniformly mean square summable. 
Using (3.23), (3.21), (3.17), and (3.10), transform $q_{0}$ in the following way:

$$
\begin{aligned}
q_{0} & =\sum_{j=0}^{\infty} R_{k j}=\sum_{j=0}^{k} R_{k j}+\sum_{j=k+1}^{\infty} R_{k j} \\
& =\left(\alpha_{k+1}+\mu^{-1}\right) \sum_{j=0}^{k}\left|Q_{k j}\right|+\left(\left(1+\mu^{-1}\right) \alpha_{k+1}+\beta_{k}\right) \sum_{j=k+1}^{\infty}\left|a_{j}\right| \\
& =\left(\alpha_{k+1}+\mu^{-1}\right) \beta_{k}+\left(\left(1+\mu^{-1}\right) \alpha_{k+1}+\beta_{k}\right) \alpha_{k+1} \\
& =\alpha_{k+1}^{2}+2 \alpha_{k+1} \beta_{k}+\mu^{-1}\left(\alpha_{k+1}^{2}+\beta_{k}\right) .
\end{aligned}
$$

Thus, if

$$
\alpha_{k+1}^{2}+2 \alpha_{k+1} \beta_{k}<d_{k+1, k+1}^{-1},
$$

then there exists a so big $\mu>0$ that condition (3.27) holds and, therefore, the solution of (3.1) is uniformly mean square summable.

Note that condition (3.30) can also be represented in the form

$$
\alpha_{k+1}<\sqrt{\beta_{k}^{2}+d_{k+1, k+1}^{-1}}-\beta_{k} .
$$

Remark 3.1. Suppose that in (3.1)

$$
a_{j}=0, \quad j>k \text {. }
$$

Then $\alpha_{k+1}=0$. So, if condition (3.32) holds and the matrix equation (3.5) has a positive semidefinite solution $D$ with $d_{k+1, k+1}>0$, then the solution of (3.1) is uniformly mean square summable.

3.2. The second way of Lyapunov functionals construction. We get another stability condition. Equation (3.1) can be represented (Step 1) in the form (2.1) with $F_{2}(t)=0$, $k=0$,

$$
F_{1}(t)=\beta x(t), \quad \beta=\sum_{j=0}^{\infty} a_{j}, \quad F_{3}(t)=-\sum_{m=1}^{[t]+r} x(t-m) \sum_{j=m}^{\infty} a_{j} .
$$

Really, calculating $\Delta F_{3}(t)$, we have

$$
\begin{aligned}
\Delta F_{3}(t) & =-\sum_{m=1}^{[t]+1+r} x(t+1-m) \sum_{j=m}^{\infty} a_{j}+\sum_{m=1}^{[t]+r} x(t-m) \sum_{j=m}^{\infty} a_{j} \\
& =-\sum_{m=0}^{[t]+r} x(t-m) \sum_{j=m+1}^{\infty} a_{j}+\sum_{m=1}^{[t]+r} x(t-m) \sum_{j=m}^{\infty} a_{j} \\
& =-x(t) \sum_{j=1}^{\infty} a_{j}+\sum_{m=1}^{[t]+r} x(t-m) a_{m} .
\end{aligned}
$$


Thus,

$$
x(t+1)=\eta(t+1)+\beta x(t)+\Delta F_{3}(t) .
$$

In this case the auxiliary equation (2.3) (Step 2) is $y(t+1)=\beta y(t)$. The function $v(t)=y^{2}(t)$ is a Lyapunov function for this equation if $|\beta|<1$. We will construct the Lyapunov functional $V(t)$ (Step 3) for (3.1) in the form $V(t)=V_{1}(t)+V_{2}(t)$, where $V_{1}(t)=\left(x(t)-F_{3}(t)\right)^{2}$. Calculating $\mathbf{E} \Delta V_{1}(t)$, by virtue of representation (3.33), we have

$$
\begin{aligned}
\mathbf{E} \Delta V_{1}(t)= & \mathbf{E}\left[\left(x(t+1)-F_{3}(t+1)\right)^{2}-\left(x(t)-F_{3}(t)\right)^{2}\right] \\
= & \mathbf{E}\left[\left(\eta(t+1)+\beta x(t)-F_{3}(t)\right)^{2}-\left(x(t)-F_{3}(t)\right)^{2}\right] \\
= & \mathbf{E}(\eta(t+1)+(\beta-1) x(t))\left(\eta(t+1)+(\beta+1) x(t)-2 F_{3}(t)\right) \\
= & \left(\beta^{2}-1\right) \mathbf{E} x^{2}(t)+\mathbf{E} \eta^{2}(t+1)+2 \beta \mathbf{E} \eta(t+1) x(t) \\
& -2 \mathbf{E} \eta(t+1) F_{3}(t)-2(\beta-1) \mathbf{E} x(t) F_{3}(t) .
\end{aligned}
$$

Using $\mu>0$, we obtain

$$
2 \mathbf{E}|\eta(t+1) x(t)| \leq \mu \mathbf{E} \eta^{2}(t+1)+\mu^{-1} \mathbf{E} x^{2}(t) .
$$

Putting

$$
B_{m}=\left|\sum_{j=m}^{\infty} a_{j}\right|, \quad \alpha=\sum_{m=1}^{\infty} B_{m}
$$

and using (3.33), (3.10), we have

$$
\begin{aligned}
2 \mathbf{E}\left|\eta(t+1) F_{3}(t)\right| & \leq 2 \sum_{m=1}^{[t]+r} B_{m} \mathbf{E}|\eta(t+1) x(t-m)| \\
& \leq \sum_{m=1}^{[t]+r} B_{m}\left(\mu \mathbf{E} \eta^{2}(t+1)+\mu^{-1} \mathbf{E} x^{2}(t-m)\right) \\
& \leq \alpha \mu \mathbf{E} \eta^{2}(t+1)+\mu^{-1} \sum_{m=1}^{[t]+r} B_{m} \mathbf{E} x^{2}(t-m), \\
2 \mathbf{E}\left|x(t) F_{3}(t)\right| & \leq 2 \sum_{m=1}^{[t]+r} B_{m} \mathbf{E}|x(t) x(t-m)| \\
& \leq \sum_{m=1}^{[t]+r} B_{m}\left(\mathbf{E} x^{2}(t)+\mathbf{E} x^{2}(t-m)\right) \\
& \leq \alpha \mathbf{E} x^{2}(t)+\sum_{m=1}^{[t]+r} B_{m} \mathbf{E} x^{2}(t-m) .
\end{aligned}
$$


As a result,

$$
\begin{aligned}
\mathbf{E} \Delta V_{1}(t) \leq & \left(\beta^{2}-1+\alpha|\beta-1|+\mu^{-1}|\beta|\right) \mathbf{E} x^{2}(t)+(1+\mu(\alpha+|\beta|)) \mathbf{E} \eta^{2}(t+1) \\
& +\left(|\beta-1|+\mu^{-1}\right) \sum_{m=1}^{[t]+r} B_{m} \mathbf{E} x^{2}(t-m) .
\end{aligned}
$$

Put now (Step 4)

$$
V_{2}(t)=\sum_{m=1}^{[t]+r} \gamma_{m} x^{2}(t-m), \quad \gamma_{m}=\left(|\beta-1|+\mu^{-1}\right) \sum_{j=m}^{\infty} B_{j} .
$$

Then, using (3.38), similar to (3.24), we have

$$
\Delta V_{2}(t)=\left(|\beta-1|+\mu^{-1}\right)\left(\alpha x^{2}(t)-\sum_{m=1}^{[t]+r} B_{m} x^{2}(t-m)\right) .
$$

So, for the functional $V(t)=V_{1}(t)+V_{2}(t)$, we obtain

$$
\begin{aligned}
\mathbf{E} \Delta V(t) \leq & \left(\beta^{2}-1+2 \alpha|\beta-1|+\mu^{-1}(\alpha+|\beta|)\right) \mathbf{E} x^{2}(t) \\
& +(1+\mu(\alpha+|\beta|)) \mathbf{E} \eta^{2}(t+1) .
\end{aligned}
$$

Thus, if

$$
\beta^{2}+2 \alpha|\beta-1|<1
$$

then there exists a so $\operatorname{big} \mu>0$ that condition $\beta^{2}+2 \alpha|\beta-1|+\mu^{-1}(\alpha+|\beta|)<1$ holds also, and, therefore, the solution of (3.1) is uniformly mean square summable.

It is easy to see that condition (3.44) can be written also in the form

$$
1+\beta>2 \alpha, \quad|\beta|<1 .
$$

\section{Particular cases}

Here, particular cases of condition (3.31) for different $k \geq 0$ are considered.

4.1. Case $k=0$. Equation (3.5) gives the solution $d_{11}=\left(1-a_{0}^{2}\right)^{-1}$, which is a positive one if $\left|a_{0}\right|<1$. From (3.17), it follows that $\beta_{0}=\left|a_{0}\right|$. Condition (3.31) takes the form

$$
\alpha_{0}<1
$$

So, under condition (4.1), the solution of (3.1) is uniformly mean square summable.

4.2. Case $k=1$. The matrix equation (3.5) is equivalent to the system of equations

$$
\begin{gathered}
a_{1}^{2} d_{22}-d_{11}=0, \\
\left(a_{1}-1\right) d_{12}+a_{0} a_{1} d_{22}=0, \\
d_{11}+2 a_{0} d_{12}+\left(a_{0}^{2}-1\right) d_{22}=-1,
\end{gathered}
$$


with the solution

$$
\begin{aligned}
& d_{11}=a_{1}^{2} d_{22}, \quad d_{12}=\frac{a_{0} a_{1}}{1-a_{1}} d_{22}, \\
& d_{22}=\frac{1-a_{1}}{\left(1+a_{1}\right)\left[\left(1-a_{1}\right)^{2}-a_{0}^{2}\right]} .
\end{aligned}
$$

The matrix $D$ is a positive semidefinite one with $d_{22}>0$ by conditions $\left|a_{1}\right|<1,\left|a_{0}\right|<$ $1-a_{1}$. Using (3.17), (4.3), we have

$$
\begin{aligned}
\beta_{1}=\left|a_{1}\right|+\left|a_{0}+\frac{d_{12}}{d_{22}}\right| & =\left|a_{1}\right|+\left|a_{0}+\frac{a_{0} a_{1}}{1-a_{1}}\right|=\left|a_{1}\right|+\frac{\left|a_{0}\right|}{1-a_{1}}, \\
d_{22}^{-1} & =1-a_{1}^{2}-a_{0}^{2} \frac{1+a_{1}}{1-a_{1}} .
\end{aligned}
$$

Condition (3.31) takes the form

$$
\alpha_{2}<\left(1-\left|a_{1}\right|\right)\left(1-\frac{\left|a_{0}\right|}{1-a_{1}}\right)
$$

Under condition (4.5), the solution of (3.1) is uniformly mean square summable.

Note that condition (4.5) can also be written in the form

$$
\alpha_{0}<1+\left|a_{0}\right| \frac{\left|a_{1}\right|-a_{1}}{1-a_{1}} .
$$

It is easy to see that condition (4.6) is not worse than (4.1). In particular, for $a_{1} \geq 0$, condition (4.6) coincides with (4.1).

4.3. Case $k=2$. The matrix equation (3.5) is equivalent to the system of equations

$$
\begin{aligned}
& a_{2}^{2} d_{33}-d_{11}=0, \\
& a_{2} d_{13}+a_{1} a_{2} d_{33}-d_{12}=0, \\
& a_{2} d_{23}+a_{0} a_{2} d_{33}-d_{13}=0, \\
& d_{11}+2 a_{1} d_{13}+a_{1}^{2} d_{33}-d_{22}=0, \\
& d_{12}+a_{0} d_{13}+a_{0} a_{1} d_{33}+\left(a_{1}-1\right) d_{23}=0, \\
& d_{22}+2 a_{0} d_{23}+\left(a_{0}^{2}-1\right) d_{33}=-1,
\end{aligned}
$$


with the solution

$$
\begin{aligned}
& d_{11}=a_{2}^{2} d_{33}, \\
& d_{12}=\frac{a_{2}\left(1-a_{1}\right)\left(a_{1}+a_{0} a_{2}\right)}{1-a_{1}-a_{2}\left(a_{0}+a_{2}\right)} d_{33}, \\
& d_{13}=\frac{a_{2}\left(a_{0}+a_{1} a_{2}\right)}{1-a_{1}-a_{2}\left(a_{0}+a_{2}\right)} d_{33}, \\
& d_{22}=\left[a_{1}^{2}+a_{2}^{2}+\frac{2 a_{1} a_{2}\left(a_{0}+a_{1} a_{2}\right)}{1-a_{1}-a_{2}\left(a_{0}+a_{2}\right)}\right] d_{33}, \\
& d_{23}=\frac{\left(a_{0}+a_{2}\right)\left(a_{1}+a_{0} a_{2}\right)}{1-a_{1}-a_{2}\left(a_{0}+a_{2}\right)} d_{33}, \\
& d_{33}=\left[1-a_{0}^{2}-a_{1}^{2}-a_{2}^{2}-2 \frac{a_{1} a_{2}\left(a_{0}+a_{1} a_{2}\right)+a_{0}\left(a_{0}+a_{2}\right)\left(a_{1}+a_{0} a_{2}\right)}{1-a_{1}-a_{2}\left(a_{0}+a_{2}\right)}\right]^{-1} .
\end{aligned}
$$

Using (3.17), (4.7), and (4.8), we have

$$
\begin{aligned}
\beta_{2} & =\left|a_{2}\right|+\left|a_{0}+\frac{d_{23}}{d_{33}}\right|+\left|a_{1}+\frac{d_{13}}{d_{33}}\right|=\left|a_{2}\right|+\frac{\left|d_{13}\right|+\left|d_{12}\right|}{\left|a_{2}\right| d_{33}} \\
& =\left|a_{2}\right|+\frac{\left|a_{0}+a_{1} a_{2}\right|+\left|\left(1-a_{1}\right)\left(a_{1}+a_{0} a_{2}\right)\right|}{\left|1-a_{1}-a_{2}\left(a_{0}+a_{2}\right)\right|} .
\end{aligned}
$$

If the matrix $D$ with the elements defined by (4.8) is a positive semidefinite one with $d_{33}>0$, then under the condition

$$
\alpha_{3}<\sqrt{\beta_{2}^{2}+d_{33}^{-1}}-\beta_{2}
$$

the solution of (3.1) is uniformly mean square summable.

\section{Examples}

Example 5.1. Consider the difference equation

$$
\begin{aligned}
x(t+1)= & \eta(t+1)+a x(t)+b x(t-1), \quad t>-1, \\
& x(\theta)=\phi(\theta), \quad \theta \in[-2,0] .
\end{aligned}
$$

From conditions (4.1) and (4.5) follow two sufficient conditions for uniformly mean square summability of the solution of (5.1):

$$
\begin{gathered}
|a|+|b|<1, \\
|a|+b<1, \quad|b|<1 .
\end{gathered}
$$

Condition (4.11) for (5.1) coincides with (5.3). Condition (3.45) takes the form

$$
1+a+b>2|b|, \quad|a+b|<1 .
$$




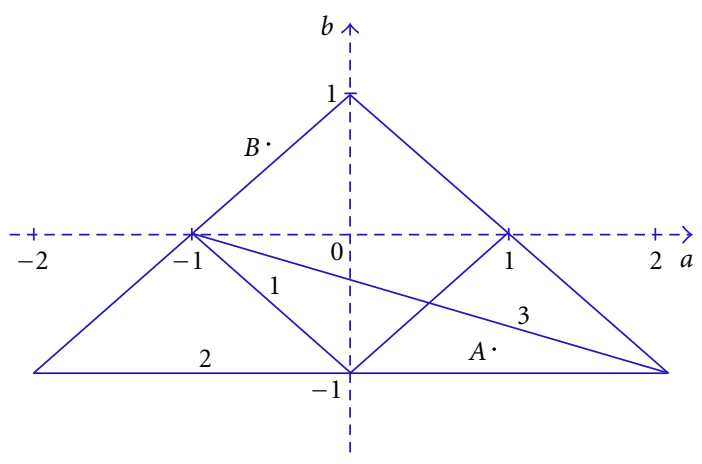

Figure 5.1

On Figure 5.1 are shown the regions of uniformly mean square summability, obtained for (5.1) by conditions (5.2) (the square number 1), (5.3) (the triangle number 2), and (5.4) (the triangle number 3).

For numerical investigation of the solution of (5.1), we determine one of the possible trajectories of the process $\eta(t), t \geq t_{0}$, in the following way. On the interval $\left[t_{0}+n h_{0}, t_{0}+\right.$ $\left.(n+1) h_{0}\right), n=0,1, \ldots$, put

$$
\eta(t)=0
$$

if

$$
t \in\left[t_{0}+n h_{0}, t_{0}+\left(n+1-\frac{1}{2^{n}}\right) h_{0}\right)
$$

or

$$
t \in\left[t_{0}+\left(n+1-\frac{1}{2^{n+1}}\right) h_{0}, t_{0}+(n+1) h_{0}\right),
$$

put

$$
\eta(t)=2^{n+2}\left(\frac{t-t_{0}}{h_{0}}-n-1+\frac{1}{2^{n}}\right)
$$

if

$$
t \in\left[t_{0}+\left(n+1-\frac{1}{2^{n}}\right) h_{0}, t_{0}+\left(n+1-\frac{3}{2^{n+2}}\right) h_{0}\right),
$$

and put

$$
\eta(t)=1-2^{n+2}\left(\frac{t-t_{0}}{h_{0}}-n-1+\frac{3}{2^{n+2}}\right)
$$




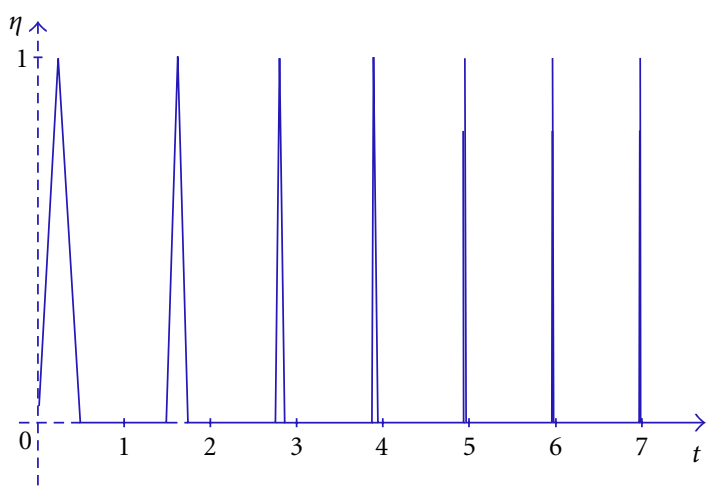

Figure 5.2

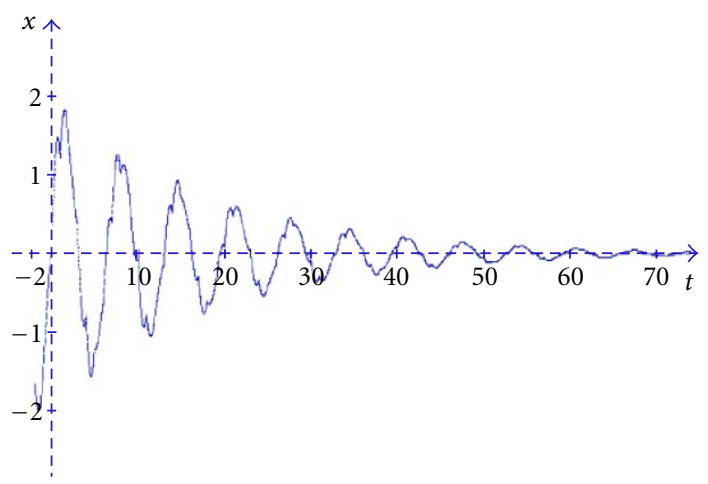

Figure 5.3

if

$$
t \in\left[t_{0}+\left(n+1-\frac{3}{2^{n+2}}\right) h_{0}, t_{0}+\left(n+1-\frac{1}{2^{n+1}}\right) h_{0}\right)
$$

The graph of the function $\eta(t)$ for $t_{0}=0, h_{0}=1$ is shown on Figure 5.2.

The function $\eta(t)$ constructed above satisfies the following conditions:

$$
0 \leq \eta(t) \leq 1, \quad \sum_{j=0}^{\infty} \eta\left(t+j h_{0}\right) \leq 1, \quad \int_{0}^{\infty} \eta(t) d t=\frac{1}{2} .
$$

It is easy to see also that for each fixed $t \in\left[t_{0}, t_{0}+h_{0}\right)$, the sequence $\eta_{j}=\eta\left(t+j h_{0}\right)$ has only one nonzero member, and therefore $\lim _{j \rightarrow \infty} \eta\left(t+j h_{0}\right)=0$. On the other hand, for every $T>0$, there exists a so large number $n$ that

$$
t_{1}=t_{0}+\left(n+1-\frac{3}{2^{n+2}}\right) h_{0}>T, \quad \eta\left(t_{1}\right)=1
$$


Therefore, $\lim _{t \rightarrow \infty} \eta(t)$ does not exist. So, the function $\eta(t)$ is an asymptotically quasitrivial function (satisfies condition (1.7)) but not an asymptotically trivial one (does not satisfy condition (1.6)).

The trajectory of (5.1) with the initial function $\phi(\theta)=\cos 2 \theta-1$ is shown in the point $A(1.1,-0.9)$, which belongs to the summability region, on Figure 5.3 with $\eta(t) \equiv 0$ and on Figure 5.4 with $\eta(t)$ described above. The trajectory of (5.1) with the initial function $\phi(\theta)=0.05 \cos 2 \theta$ is shown in the point $B(-0.5,0.6)$, which does not belong to the summability region, on Figure 5.5 with $\eta(t) \equiv 0$ and on Figure 5.6 with $\eta(t)$ described above. The points $A$ and $B$ are shown on Figure 5.1.

Example 5.2. Consider the difference equation

$$
\begin{gathered}
x(t+1)=\eta(t+1)+a x(t)+\sum_{j=1}^{[t]+r} b^{j} x(t-j), \quad t>-1, \\
x(\theta)=\phi(\theta), \quad \theta \in[-(r+1), 0], \quad r \geq 0 .
\end{gathered}
$$

From condition (4.1), it follows that the inequality

$$
|a|<\frac{1-2|b|}{1-|b|}, \quad|b|<\frac{1}{2}
$$

is a sufficient condition for uniformly mean square summability of the solution of (5.14). Condition (4.5) gives us a sufficient condition for uniformly mean square summability of the solution of (5.14) in the form

$$
|a|<\left(\frac{1-2|b|}{1-|b|}\right)\left(\frac{1-b}{1-|b|}\right), \quad|b|<\frac{1}{2} .
$$

From (4.8), (4.10), and (4.11), we obtain another sufficient condition for uniformly mean square summability of the solution of (5.14):

$$
\begin{gathered}
\frac{|b|^{3}}{1-|b|}<\sqrt{\beta_{2}^{2}+d_{33}^{-1}}-\beta_{2}, \quad|b|<1, \\
\beta_{2}=b^{2}+\frac{\left|a+b^{3}\right|+(1-b)|b(1+a b)|}{\left|1-b-b^{2}\left(a+b^{2}\right)\right|}, \\
d_{33}^{-1}=1-a^{2}-b^{2}-b^{4}-2 b \frac{b^{2}\left(a+b^{3}\right)+a\left(a+b^{2}\right)(1+a b)}{1-b-b^{2}\left(a+b^{2}\right)} .
\end{gathered}
$$

Using Mathematica program for solution of the matrix equation (3.5), sufficient condition (3.31) for uniformly mean square summability of the solution of (5.14) was obtained also for $k=3$ and $k=4$. In particular, for $k=3$, this condition takes the form

$$
\begin{gathered}
\frac{b^{4}}{1-|b|}<\sqrt{\beta_{3}^{2}+d_{44}^{-1}}-\beta_{3}, \quad|b|<1, \\
\beta_{3}=\left|b^{3}\right|+\left|a+\frac{d_{34}}{d_{44}}\right|+\left|b+\frac{d_{24}}{d_{44}}\right|+\left|b^{2}+\frac{d_{14}}{d_{44}}\right|,
\end{gathered}
$$




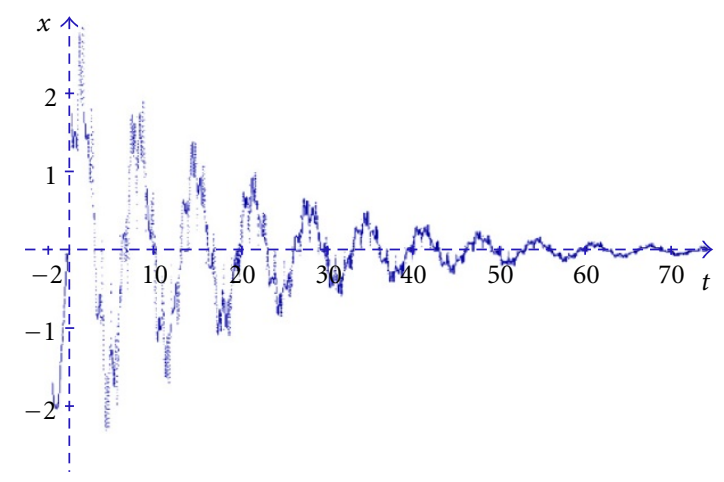

Figure 5.4

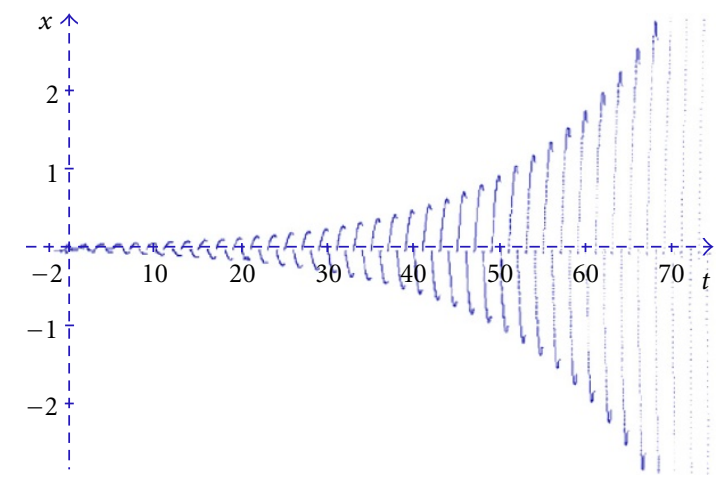

Figure 5.5

where

$$
\begin{aligned}
& \frac{d_{14}}{d_{44}}=b^{3}\left[b^{3}+b^{5}-b^{8}+a\left(1-b^{3}+b^{4}\right)\right] G^{-1}, \\
& \frac{d_{24}}{d_{44}}=b^{2}\left[a^{2} b+b^{2}+b^{5}-b^{6}-b^{8}+a\left(1+b^{4}+b^{6}\right)\right] G^{-1}, \\
& \frac{d_{34}}{d_{44}}=b\left[b^{2}+a^{3} b^{2}+b^{4}-b^{7}+a^{2}\left(b+b^{4}\right)+a\left(1+2 b^{3}+b^{5}-b^{6}-b^{8}\right)\right] G^{-1}, \\
& \begin{aligned}
& d_{44}=G {\left[1-b-b^{2}-a^{4} b^{3}-2 b^{4}+2 b^{7}-2 b^{8}+2 b^{9}-b^{10}-b^{12}+b^{13}\right.} \\
& \quad-b^{14}+b^{17}-a^{3}\left(b^{2}+b^{5}\right)-a^{2}\left(1+b+5 b^{4}-b^{5}+b^{6}-2 b^{7}-b^{9}\right) \\
&\left.\quad-a b^{2}\left(1+4 b-b^{2}+5 b^{3}-b^{4}+b^{5}-4 b^{6}+4 b^{7}-b^{10}+b^{11}\right)\right]^{-1}, \\
& G=1-b-a b^{2}-\left(1+a^{2}\right) b^{3}-b^{4}-a b^{5}-b^{6}+b^{7}+b^{9} .
\end{aligned}
\end{aligned}
$$




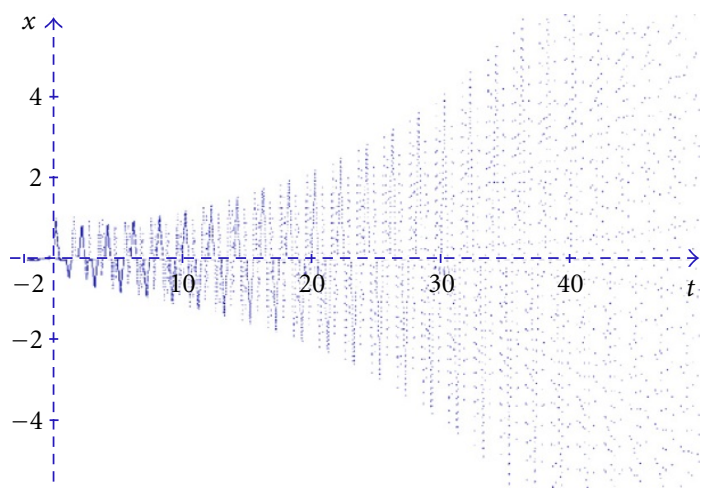

Figure 5.6

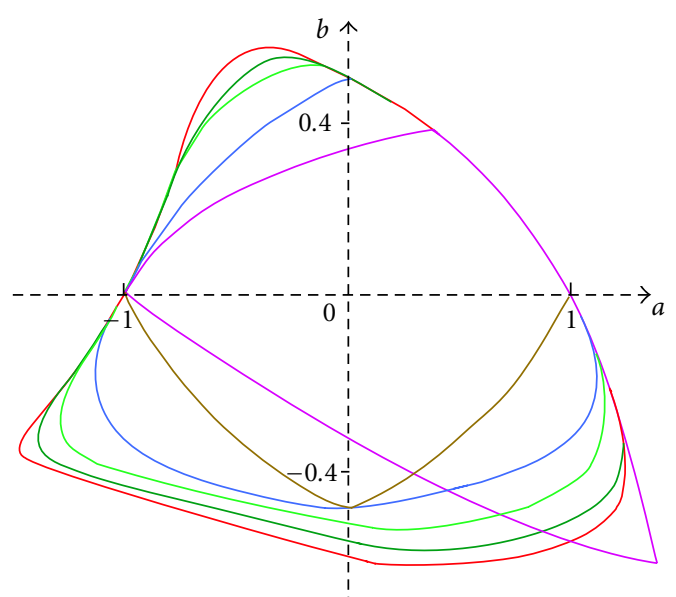

Figure 5.7

Condition (3.45) for (5.14) takes the form

$$
-\frac{1-3|b|}{(1-b)(1-|b|)}<a<\frac{1-2 b}{1-b}, \quad|b|<1 .
$$

On Figure 5.7, the regions of uniformly mean square summability of the solution of (5.14) are shown, obtained by condition (3.31). For $k=0$ (condition (5.15), the brown curve), for $k=1$ (condition (5.16), the blue curve), for $k=2$ (condition (5.17), the green curve), for $k=3$ (condition (5.18), the cyan curve), for $k=4$ (the red curve) and also obtained by condition (5.20) (the magenta curve).

As it is shown on Figure 5.7 (and naturally it can be shown analytically), for $b \geq 0$ condition (5.15) coincides with condition (5.16) and, for $a \geq 0, b \geq 0$, conditions (5.15), (5.16), (5.17), and (5.18) give the same region of uniformly mean square summability, 


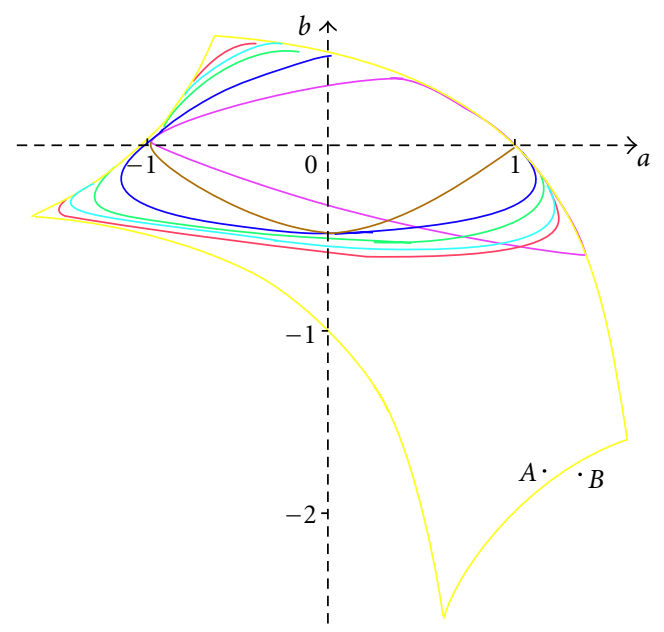

Figure 5.8

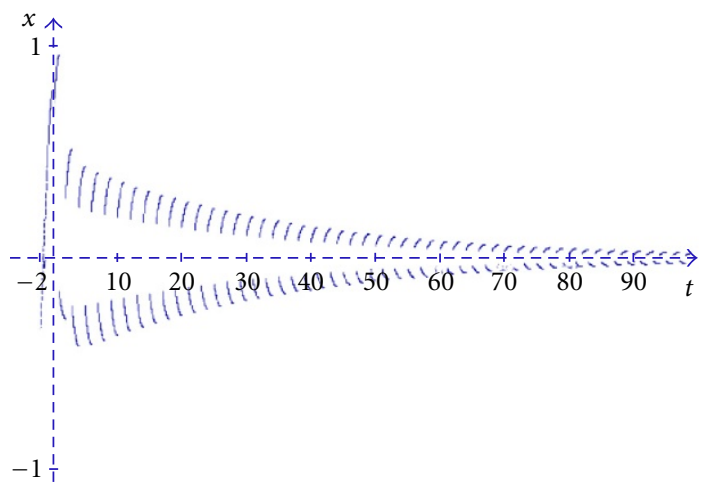

Figure 5.9

which is defined by the inequality

$$
a+\frac{b}{1-b}<1, \quad b<1
$$

Note also that the region of uniformly mean square summability $Q_{k}$ of the solution of (5.14), obtained by condition (3.31), expands if $k$ increases, that is, $Q_{0} \subset Q_{1} \subset Q_{2} \subset$ $Q_{3} \subset Q_{4}$. So, to get a greater region of uniformly mean square summability, one can use condition (3.31) for $k=5, k=6$, and so forth. But it is clear that each region $Q_{k}$ can be obtained by the condition $|b|<1$ only. 


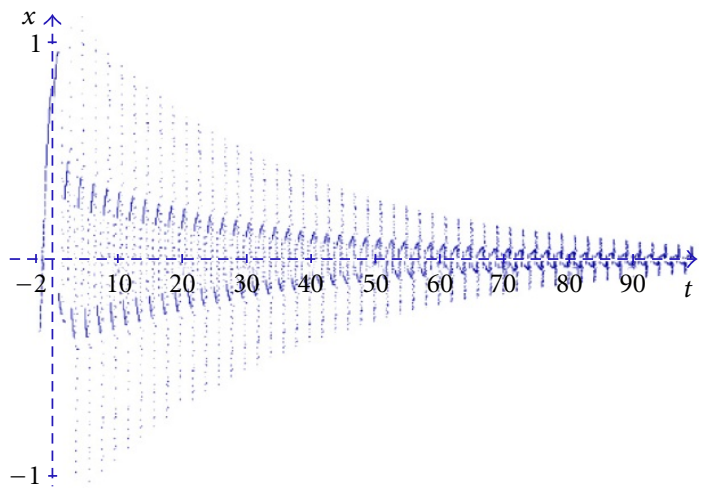

Figure 5.10

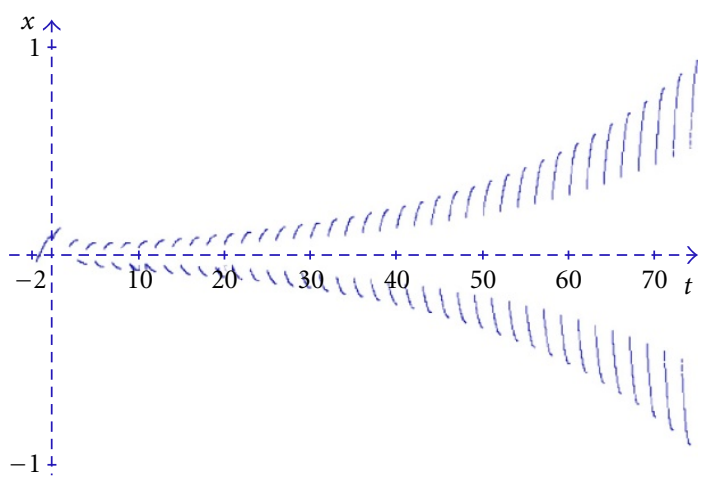

Figure 5.11

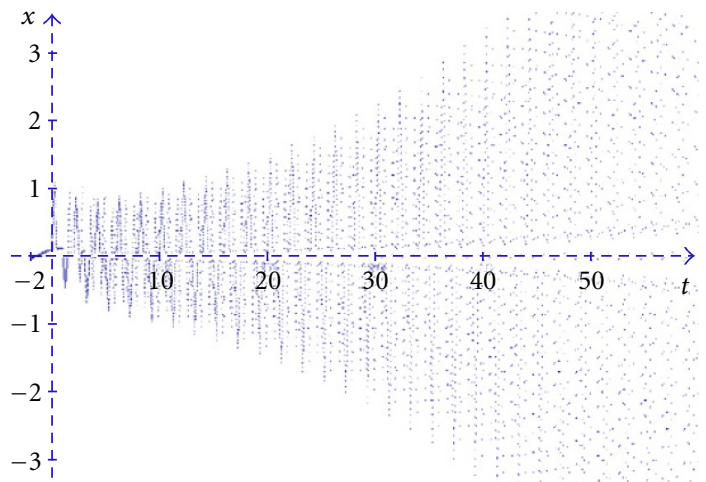

Figure 5.12 
To obtain a condition of another type for uniformly mean square summability of the solution of (5.14), transform the sum from (5.14) for $t>0$ in the following way:

$$
\begin{aligned}
\sum_{j=1}^{[t]+r} b^{j} x(t-j) & =b \sum_{j=1}^{[t]+r} b^{j-1} x(t-j) \\
& =b \sum_{j=0}^{[t]-1+r} b^{j} x(t-1-j) \\
& =b\left(x(t-1)+\sum_{j=1}^{[t]-1+r} b^{j} x(t-1-j)\right) \\
& =b[(1-a) x(t-1)+x(t)-\eta(t)] .
\end{aligned}
$$

Substituting (5.22) into (5.14), we obtain (5.14) in the form

$$
\begin{gathered}
x(t+1)=\eta(t+1)+a x(t)+\sum_{j=1}^{r-1} b^{j} x(t-j), \quad t \in(-1,0], \\
x(t+1)=\eta_{1}(t+1)+(a+b) x(t)+b(1-a) x(t-1), \\
\eta_{1}(t+1)=\eta(t+1)-b \eta(t), \quad t>0 .
\end{gathered}
$$

The corresponding matrix $D$ is defined by (4.3) with $a_{0}=a+b, a_{1}=b(1-a)$, and it is a positive semidefinite one if and only if

$$
|b(1-a)|<1, \quad|a+b|<1-b(1-a) .
$$

On Figure 5.8 the graph on Figure 5.7 is shown together with the region of uniformly mean square summability obtained by condition (5.24) (the yellow curve).

The trajectory of (5.14) with $r=1$ and the initial functional $\phi(\theta)=0.8 \cos \theta$ is shown in the point $A(1.2,-1.8)$, which belongs to the summability region, on Figure 5.9 with $\eta(t) \equiv 0$ and on Figure 5.10 with $\eta(t)$ described above. The trajectory of (5.14) with $r=1$ and the initial functional $\phi(\theta)=0.1 \cos \theta$ is shown in the point $B(1.33,-1.8)$, which does not belong to the summability region, on Figure 5.11 with $\eta(t) \equiv 0$ and on Figure 5.12 with $\eta(t)$ described above. The points $A$ and $B$ are shown on Figure 5.8.

\section{References}

[1] M. G. Blizorukov, On the construction of solutions of linear difference systems with continuous time, Differ. Uravn. 32 (1996), no. 1, 127-128, (Russian), translated in Differential Equations 32 (1996), no. 1, 133-134.

[2] V. B. Kolmanovskii, The stability of certain discrete-time Volterra equations, J. Appl. Math. Mech. 63 (1999), no. 4, 537-543.

[3] V. B. Kolmanovskii and A. Myshkis, Applied Theory of Functional-Differential Equations, Mathematics and Its Applications (Soviet Series), vol. 85, Kluwer Academic Publishers, Dordrecht, 1992.

[4] V. B. Kolmanovskiĭ and V. R. Nosov, Stability of Functional-Differential Equations, Mathematics in Science and Engineering, vol. 180, Academic Press, London, 1986. 
[5] V. B. Kolmanovskii and L. E. Shaikhet, New results in stability theory for stochastic functionaldifferential equations (SFDEs) and their applications, Proceedings of Dynamic Systems and Applications, Vol. 1 (Atlanta, Ga, 1993), Dynamic Publishers, Georgia, 1994, pp. 167-171.

[6] General method of Lyapunov functionals construction for stability investigation of stochastic difference equations, Dynamical Systems and Applications, World Sci. Ser. Appl. Anal., vol. 4, World Scientific Publishing, New Jersey, 1995, pp. 397-439.

[7] A method for constructing Lyapunov functionals for stochastic differential equations of neutral type, Differ. Uravn. 31 (1995), no. 11, 1851-1857 (Russian), translated in Differential Equations 31 (1996), no. 11, 1819-1825.

[8] Control of Systems with Aftereffect, Translations of Mathematical Monographs, vol. 157, American Mathematical Society, Rhode Island, 1996.

[9] - Matrix Riccati equations and stability of stochastic linear systems with nonincreasing delays, Funct. Differ. Equ. 4 (1997), no. 3-4, 279-293.

[10] Riccati equations and stability of stochastic linear systems with distributed delay, Advances in Systems, Signals, Control and Computers (V. Bajic, ed.), vol. 1, IAAMSAD and SA branch of the Academy of Nonlinear Sciences, Durban, 1998, pp. 97-100.

[11] Construction of Lyapunov functionals for stochastic hereditary systems: a survey of some recent results, Math. Comput. Modelling 36 (2002), no. 6, 691-716.

[12] Some peculiarities of the general method of Lyapunov functionals construction, Appl. Math. Lett. 15 (2002), no. 3, 355-360.

[13] About one application of the general method of Lyapunov functionals construction, International Journal of Robust and Nonlinear Control 13 (2003), no. 9, 805-818.

[14] D. G. Korenevskiü, Criteria for the stability of systems of linear deterministic and stochastic difference equations with continuous time and with delay, Mat. Zametki 70 (2001), no. 2, 213-229 (Russian), translated in Math. Notes 70 (2001), no. 1-2, 192-205.

[15] H. Péics, Representation of solutions of difference equations with continuous time, Proceedings of the 6th Colloquium on the Qualitative Theory of Differential Equations (Szeged, 1999), Proc. Colloq. Qual. Theory Differ. Equ., no. 21, Electron. J. Qual. Theory Differ. Equ., Szeged, 2000, pp. 1-8.

[16] G. P. Pelyukh, Representation of solutions of difference equations with a continuous argument, Differ. Uravn. 32 (1996), no. 2, 256-264 (Russian), translated in Differential Equations 32 (1996), no. 2, 260-268.

[17] L. E. Shaikhet, Stability in probability of nonlinear stochastic hereditary systems, Dynam. Systems Appl. 4 (1995), no. 2, 199-204.

[18] Modern state and development perspectives of Lyapunov functionals method in the stability theory of stochastic hereditary systems, Theory of Stochastic Processes 2(18) (1996), no. 1-2, 248-259.

[19] _ Necessary and sufficient conditions of asymptotic mean square stability for stochastic linear difference equations, Appl. Math. Lett. 10 (1997), no. 3, 111-115.

[20] A. N. Sharkovsky and Yu. L. Maĭstrenko, Difference equations with continuous time as mathematical models of the structure emergences, Dynamical Systems and Environmental Models (Eisenach, 1986), Math. Ecol., Akademie-Verlag, Berlin, 1987, pp. 40-49.

[21] V. Volterra, Leçons sur la Théorie Mathématique de la Lutte pour la Vie, Gauthier-Villars, Paris, 1931.

Leonid Shaikhet: Department of Mathematics, Informatics, and Computing, Donetsk State Academy of Management, 163a Chelyuskintsev street, Donetsk 83015, Ukraine

E-mail address: leonid@dsam.donetsk.ua 